\title{
Universal bounds for asset prices in heterogeneous economies
}

\section{Journal Article}

\section{Author(s):}

Malamud, Semyon

Publication date:

2008

Permanent link:

https://doi.org/10.3929/ethz-b-000010981

Rights / license:

In Copyright - Non-Commercial Use Permitted

Originally published in:

Finance and Stochastics 12(3), https://doi.org/10.1007/s00780-008-0062-z 


\title{
Universal bounds for asset prices in heterogeneous economies
}

\author{
Semyon Malamud
}

Received: 17 July 2007 / Accepted: 18 January 2008 / Published online: 5 April 2008

(C) Springer-Verlag 2008

\begin{abstract}
We establish universal bounds for asset prices in heterogeneous complete market economies with scale invariant preferences. Namely, for each agent in the economy we consider an artificial homogeneous economy populated solely by this agent, and calculate the "homogeneous" price of an asset in each of these economies. Dumas (Rev. Financ. Stud. 2, 157-188, 1989) conjectured that the risk free rate in a heterogeneous economy must lie in the interval determined by the minimal and maximal of the "homogeneous" risk free rates. We show that the answer depends on the risk aversions of the agents in the economy: the upper bound holds when all risk aversions are smaller than one, and the lower bound holds when all risk aversions are larger than one. The bounds almost never hold simultaneously. Furthermore, we prove these bounds for arbitrary assets.
\end{abstract}

Keywords Heterogeneity $\cdot$ Asset prices $\cdot$ Yield curve $\cdot$ Bounds

Mathematics Subject Classification (2000) 91B28 · 91B60 -91B64 · 91B70

JEL Classification D91 $\cdot$ E43 $\cdot$ G12

\section{Introduction}

In a heterogeneous economy, the wealth fluctuates randomly between different agents. Obviously, an agent who is rich in a particular state will have a large influence on asset prices in that state. For example, if this agent owns almost all goods in the economy, the asset prices will be very close to the prices that would prevail in a homogeneous economy populated by this single agent. Thus, it is natural that these

S. Malamud ( $₫)$

ETH Zürich, D-MATH, Rämistrasse 101, 8092 Zürich, Switzerland

e-mail: semka@math.ethz.ch 
"homogeneous" prices in the artificial economies populated by one or another single agent play an important role in determining the behavior of asset prices.

In a production economy with two agents, Dumas [6] conjectured that the risk free rate in a heterogeneous economy should lie between the maximal and the minimal "homogeneous" risk free rates of the artificial economies. Wang [13] considered a continuous time version of our economy with a geometric Brownian motion aggregate endowment and two agents having identical discount factors and risk aversions 1 and 0.5 . He showed by numerical simulations that the bounds conjectured by Dumas [6] do not hold.

In this paper we correct and resolve this conjecture for a general heterogeneous economy with CRRA preferences. Our results can be directly extended to any scale invariant preferences, including heterogeneous beliefs (such as Wang [11, 12]) and state dependent preferences generated by habit formation (such as, e.g., Constantinides [5] and Abel [1]). Even though we work in discrete time, all our results literally hold for continuous time economies.

In [9], we developed a tool that makes it possible to analyze many aspects of general heterogeneous economies. Precisely, we construct an explicit "aggregator" function of as many variables as there are agents. The equilibrium state price density of the heterogeneous economy is equal to the aggregator function evaluated at the state price densities of artificial homogeneous economies populated by exactly one agent from the heterogeneous economy. Analytic properties of the aggregator function translate directly into economic mechanisms. The main technical result of this paper is the observation that the aggregator function is convex (concave) when all risk aversions are smaller (larger) than one. This important property corrects and resolves the conjecture of Dumas [6].

There are many papers that investigate the influence of heterogeneity on asset prices. See, e.g., Wang [11, 12], Gollier and Zeckhauser [7].

A special case of our model is considered by Wang [13] in a continuous time setting. He examined the case of two agents with identical discount factors and very special choices of risk aversion and studied Pareto-efficient allocations.

Dumas [6] numerically analyzed a model closely related to ours (and, of course, the model of Wang [13]). He simulated a two agent production economy and obtained a detailed description of consumption, portfolio and wealth allocations and their intertemporal dynamics. He also studied the behavior of short term risk free rates and discovered several interesting properties of those.

Chan and Kogan [3] analyzed a similar model with heterogeneous risk aversion and habit formation, but only when heterogeneity is small (weak), and used perturbation theory to determine the status of several stylized facts.

Lengwiler [8] considered an economy with heterogeneous discount factors. Benninga and Mayshar [2] investigated a one-period economy with heterogeneous risk aversions and discount factors and showed that the conventional representative agent has a decreasing risk aversion. They studied implications of this phenomenon for option pricing. Gollier and Zeckhauser [7] analyzed Pareto-efficient allocations in heterogeneous economies with general utility functions and discovered some interesting aggregation phenomena. There are also aggregation results (see $[4,10])$ justifying the conventional representative agent approach. 


\section{The model}

The model is identical to that of [9], Sect. 2. We briefly recall the setup for the reader's convenience.

We assume a discrete time, pure exchange economy with a single perishable consumption good (numeraire). The aggregate endowment stream $W_{t}, t=0, \ldots, T$, equivalently, the single good stream, is an arbitrary, positive process. The stream is normalized by $W_{0}=1$. The information structure is encoded in the filtration $\left(\mathcal{F}_{t}, t=0, \ldots, T\right)$ on the underlying probability space $(\Omega, \mathcal{B}, P)$ and generated by the aggregate endowment process $\left(W_{t}\right)$.

The economy is populated by $n$ CRRA agents labeled by $i, i=1, \ldots, n$. We denote by $N=\{1, \ldots, n\}$ the set of all agents.

Agent $i$ has a constant relative risk aversion (CRRA) utility function. He chooses his random consumption $x_{i t}$ at each time $t=0, \ldots, T$ and in each possible state of the world to maximize his expected discounted intertemporal utility

$$
E\left[\sum_{t=0}^{T} \delta_{i}^{t} \frac{x_{i t}^{1-\gamma_{i}}-1}{1-\gamma_{i}}\right] .
$$

Here, $\gamma_{i}$ is the relative risk aversion of agent $i$ and $\delta_{i}$ is his discount factor (patience). Agent $i$ is endowed with the fraction $\eta_{i}$ of the aggregate endowment and $\sum_{i=1}^{n} \eta_{i}=1$.

\section{Market equilibrium and state price densities}

We assume that the market is dynamically complete. In this case, equilibrium allocations are Pareto-efficient and are uniquely determined by specifying the initial consumption $x_{i 0}$ for each agent $i$. Furthermore, any equilibrium can be characterized as an Arrow-Debreu equilibrium via the unique (because of market completeness) state price density process $\mathbf{M}=\left(M_{t}\right)$. See also [9]. The corresponding equilibrium equations for the state price densities take the form

$$
\sum_{i=1}^{n} x_{i t}=W_{t}
$$

for all $t=1, \ldots, T$ (equilibrium market clearing at time zero follows from Walras' law), with the equilibrium optimal consumption

$$
x_{i t}=\delta_{i}^{t b_{i}} M_{t}^{-b_{i}} x_{i 0}
$$

Here, $b_{i}$ is the reciprocal of relative risk aversion, $b_{i}=1 / \gamma_{i}$. It is usually referred to as cautiousness.

In the homogeneous economy populated by a single agent with parameters $\left(\delta_{i}, \gamma_{i}\right)$, state price densities $M_{t}$ are given by $\delta_{i}^{t} W_{t}^{-\gamma_{i}}$ (this follows directly from (3.1)). When agents are heterogeneous, state price densities change over time and fluctuate between the "representative" state prices $\delta_{i}^{t} W_{t}^{-\gamma_{i}}$. A similar phenomenon takes place 
for all asset prices. Basing on extensive numerical analysis, Dumas [6] conjectured that risk free rates in a heterogeneous production economy must stay within the interval between the minimal and the maximal "representative" risk free rates. Wang [13] has shown that this is not true in a two agent continuous time analogue of our economy.

As mentioned in the introduction, the relationship between equilibrium state price densities and the representative state price densities is determined by the aggregator function, constructed in [9]. We recall this representation (Proposition 4.1 in [9]), including new, simple, but important bounds for the aggregator function.

Proposition 3.1 The $\tau$-period stochastic discount factor $M_{t+\tau} / M_{t}$ satisfies

$$
\frac{M_{t+\tau}}{M_{t}}=F_{t}\left(\delta_{1}^{\tau}\left(\frac{W_{t+\tau}}{W_{t}}\right)^{-\gamma_{1}}, \ldots, \delta_{n}^{\tau}\left(\frac{W_{t+\tau}}{W_{t}}\right)^{-\gamma_{n}}\right),
$$

where $F_{t}=F_{t}\left(y_{1}, \ldots, y_{n}\right)$ is the unique solution to

$$
\sum_{i=1}^{n} F_{t}^{-b_{i}} y_{i}^{b_{i}}\left(x_{i t} W_{t}^{-1}\right)=1
$$

The aggregator function $F_{t}$ satisfies the inequality $\max _{i} y_{i} \geq F_{t} \geq \min _{i} y_{i}$.

Proof The representation for $M_{t}$ is a direct consequence of the equilibrium equations (3.1); see [9], Proposition 4.1 for a proof. It remains to prove the bounds. For simplicity, we do this only for $F_{0}=F$. Suppose that $F>\max _{i} y_{i}$. Then we get a contradiction from

$$
1=\sum_{i=1}^{n} F^{-b_{i}} y_{i}^{b_{i}} x_{i 0}<\sum_{i=1}^{n}\left(y_{i} / \max _{i} y_{i}\right)^{b_{i}} x_{i 0} \leq \sum_{i=1}^{n} x_{i 0}=1,
$$

where the last identity is the equilibrium market clearing at time zero. The second bound is proved similarly.

By the definition of state price densities, under the standard no bubble condition, the price $P_{t}^{\mathbf{D}}$ at time $t$ of an asset with dividend process $\mathbf{D}=\left(D_{t}, t=1, \ldots, T\right)$ is given by

$$
P_{t}^{\mathbf{D}}=E_{t}\left[\sum_{\tau=1}^{T-t} \frac{M_{t+\tau}}{M_{t}} D_{t+\tau}\right]
$$

\section{Convexity and concavity of the aggregator function}

Definition 4.1 Let $H \subset \mathbb{R}^{n}$ be a convex set. A function $f: H \rightarrow \mathbb{R}$ is called affine linear if

$$
\sum_{i=1}^{m} \lambda_{i} f\left(h_{i}\right)=f\left(\sum_{i} \lambda_{i} h_{i}\right)
$$


for any $m \geq 1$, vectors $h_{i} \in H, i=1, \ldots, m$ and weights $\lambda_{i}>0, i=1, \ldots, m$ with $\sum_{i} \lambda_{i}=1$.

It is easy to see that if $F=\sum_{i=1}^{\ell} f_{i}$ is affine linear on $H$ and each $f_{i}$ is convex, then each $f_{i}$ is itself affine linear on $H$.

Lemma 4.2 Let $f: \mathbb{R}^{n} \rightarrow \mathbb{R}$ be a convex function and $X=\left(X_{1}, \ldots, X_{n}\right)$ a random vector $\Omega \rightarrow \mathbb{R}^{n}$. Suppose also that $(\Omega, \mathcal{B})$ is separable. Let

$$
\operatorname{supp}(X)=\left\{h \in \mathbb{R}^{n}: \operatorname{essinf}\|X-h\|=0\right\}
$$

be the essential image of the vector variable $X$ (that is, the minimal support of its distribution). Suppose that

$$
E[X]=E\left[f\left(X_{1}, \ldots, X_{n}\right)\right]=f\left(E\left[X_{1}\right], \ldots, E\left[X_{n}\right]\right)=f(E[X]) .
$$

Then the function $f$ is affine linear on the closed convex hull of $\operatorname{supp}(X)$.

Proof The following fact is well known: if

$$
f\left(\lambda_{0} h_{0}+\cdots+\lambda_{\ell} h_{\ell}\right)=\sum_{i=0}^{\ell} \lambda_{i} f\left(h_{i}\right)
$$

for some weights $\lambda_{k}>0, \sum_{k=0}^{\ell} \lambda_{k}=1$ and vectors $h_{0}, \ldots, h_{\ell} \in \mathbb{R}^{n}$, then the function $f$ is affine linear on the convex hull of the vectors $h_{0}, \ldots, h_{\ell}$.

Let $\left\{A_{k}\right\}_{k=1,2, \ldots}$ be a sequence of subsets generating $\mathcal{B}$. Consider the finite $\sigma$-algebras $\Omega_{\ell}$ generated by $A_{k}, k \leq \ell$. Then, applying Jensen's inequality twice, we get

$$
E[f(X)]=E\left[E\left[f(X) \mid \Omega_{\ell}\right]\right] \geq E\left[f\left(E\left[X \mid \Omega_{\ell}\right]\right)\right] \geq f(E[X])
$$

and (4.1) implies that

$$
E\left[f\left(E\left[X \mid \Omega_{\ell}\right]\right)\right]=f(E[X])
$$

Now, the random variable $E\left[X \mid \Omega_{\ell}\right]$ takes only a finite number of values, and therefore the above fact implies that $f$ is affine linear on the convex hull of $\operatorname{supp}\left(E\left[X \mid \Omega_{\ell}\right]\right)$. The claim follows by passing to the limit.

We will also need

Lemma 4.3 Let $F\left(x_{1}, \ldots, x_{n}, y\right): \mathbb{R}_{+}^{n+1} \rightarrow \mathbb{R}$ be a differentiable convex (concave) function. Suppose that for each $\left(x_{1}, \ldots, x_{n}\right)$ there exists a unique solution $y=y\left(x_{1}, \ldots, x_{n}\right)$ to the equation

$$
F\left(x_{1}, \ldots, x_{n}, y\right)=0
$$

and that

$$
\left.\frac{\partial F}{\partial y}\right|_{\left(x_{1}, \ldots, x_{n}, y\left(x_{1}, \ldots, x_{n}\right)\right)}<0
$$


for all $\left(x_{1}, \ldots, x_{n}\right) \in \mathbb{R}_{+}^{n}$. Then

(1) the function $y: R_{+}^{n} \rightarrow \mathbb{R}_{+}$is convex (concave);

(2) if the function $y$ is affine linear on a convex set $H \subset \mathbb{R}^{n}$, then the function $F$ is affine linear on the convex hull of the set

$$
H_{1}:=\{(x, y(x)) \mid x \in H\}
$$

Proof (1) Let $F$ be convex. Suppose that the function $y\left(x_{1}, \ldots, x_{n}\right)$ is not convex. Then there exist vectors $h_{1}, h_{2} \in \mathbb{R}^{n}$ such that

$$
y\left(\left(h_{1}+h_{2}\right) / 2\right)>\left(y\left(h_{1}\right)+y\left(h_{2}\right)\right) / 2 .
$$

Note that, since $F$ is convex, the derivative $\partial F / \partial y$ increases in $y$ and therefore, by the assumption made, we have

$$
\frac{\partial F}{\partial y}<0
$$

if $y \leq y\left(x_{1}, \ldots, x_{n}\right)$. In particular, the function $g(y)=F\left(\left(h_{1}+h_{2}\right) / 2, y\right)$ decreases in $y$ on the segment $\left(0, y\left(\left(h_{1}+h_{2}\right) / 2\right)\right.$. Therefore, using the definition of the function $y\left(x_{1}, \ldots, x_{n}\right)$, convexity of $F$ and monotonicity of $F$ in $y$, we arrive at

$$
\begin{aligned}
0 & =\frac{1}{2} F\left(h_{1}, y\left(h_{1}\right)\right)+\frac{1}{2} F\left(h_{2}, y\left(h_{2}\right)\right) \\
& >F\left(\left(h_{1}+h_{2}\right) / 2,\left(y\left(h_{1}\right)+y\left(h_{2}\right)\right) / 2\right) \\
& >F\left(\left(h_{1}+h_{2}\right) / 2, y\left(\left(h_{1}+h_{2}\right) / 2\right)\right)=0 .
\end{aligned}
$$

Thus, we have reached a contradiction. The proof of the case when $F$ is concave is completely identical; all inequality signs are simply reversed.

(2) Let $h_{1}, \ldots, h_{m} \in H$, and let $\lambda_{1}, \ldots, \lambda_{m}>0$ be any weights, $\sum_{i} \lambda_{i}=1$. We have

$$
\begin{aligned}
0 & =\sum_{i=1}^{m} \lambda_{m} F\left(h_{m}, y\left(h_{m}\right)\right) \\
& =F\left(\sum_{i=1}^{m} \lambda_{m} h_{m}, y\left(\sum_{i=1}^{m} \lambda_{m} h_{m}\right)\right)=F\left(\sum_{i=1}^{m} \lambda_{m}\left(h_{m}, y\left(h_{m}\right)\right)\right),
\end{aligned}
$$

since $y$ is affine linear on $H$. The claim follows from Lemma 4.2.

Lemma 4.4 The function $f(M, a):=M^{1-b} a^{b}: \mathbb{R}_{+}^{2} \rightarrow \mathbb{R}$ is convex if $b \geq 1$ and concave if $0 \leq b \leq 1$. If $b \neq 0,1$, then the function $f(M, a)$ is affine linear on the segment $\left(M_{1}, a_{1}\right)+t\left(M_{2}-M_{1}, a_{2}-a_{1}\right), t \in[0,1]$, between the points $\left(M_{1}, a_{1}\right)$ and $\left(M_{2}, a_{2}\right)$ if and only if $M_{1} a_{1}^{-1}=M_{2} a_{2}^{-1}$. 
Proof A smooth function is convex (concave) if and only if its Hessian is nonnegative (nonpositive) definite. We have

$$
H(f):=\left(\begin{array}{cc}
f_{M M} & f_{a M} \\
f_{M a} & f_{a a}
\end{array}\right)=M^{1-b} a^{b} b(b-1)\left(\begin{array}{cc}
M^{-2} & -M^{-1} a^{-1} \\
-M^{-1} a^{-1} & a^{-2}
\end{array}\right) .
$$

The matrix

$$
\left(\begin{array}{cc}
M^{-2} & -M^{-1} a^{-1} \\
-M^{-1} a^{-1} & a^{-2}
\end{array}\right)
$$

is nonnegative definite, and the claim follows, since $b(b-1) \geq 0$ for $b \geq 1$ and $\leq 0$ for $b \in[0,1]$. As for the second statement, the function is affine linear if and only if its second derivative with respect to $t$ vanishes. By (4.6), this means that

$$
M a^{-1}=\left(M_{2}-M_{1}\right)\left(a_{2}-a_{1}\right)^{-1}
$$

for any point $(M, a)$ on the segment. The claim follows.

Proposition 4.5 Let $F_{t}$ be the aggregator function defined in Proposition 3.1. Then

(1) If $\gamma_{i} \leq 1$ for all $i$ then $F_{t}: \mathbb{R}_{+}^{n} \rightarrow \mathbb{R}_{+}$is a convex function.

(2) If $\gamma_{i} \geq 1$ for all $i$ then $F_{t}$ is a concave function.

Proof For simplicity, let $t=0$. Consider the function

$$
G\left(a_{1}, \ldots, a_{n}, M\right)=\sum_{i \in N} M^{1-b_{i}} a_{i}^{b_{i}} x_{i 0}-M .
$$

Recall that $b_{i}=\gamma_{i}^{-1}$. It follows from Proposition 3.1 that $G\left(a_{1}, \ldots, a_{n}, M\right)=0$ if and only if $M=F_{0}\left(a_{1}, \ldots, a_{n}\right)$, where $F_{0}$ is the aggregator function.

(1) The function $f_{i}\left(M, a_{i}\right):=M^{1-b_{i}} a_{i}^{b_{i}}$ is convex for each $i$ by Lemma 4.4. As a sum of convex functions, $G$ is hence again convex. Since $\gamma_{i} \leq 1$ for all $i, b_{i} \geq 1$, and therefore $\frac{\partial G}{\partial M}<0$. By Lemma $4.3, F_{0}$ is convex.

(2) In the case where $\gamma_{i} \geq 1$ for all $i$ (i.e., $b_{i} \leq 1$ ), the function $G$ is concave by Lemma 4.4. Note that $G$ is not globally decreasing, but it fulfills the weaker monotonicity assumption of Lemma 4.3. In fact, we have

$$
\frac{\partial G}{\partial M}=\sum_{i \in N}\left(1-b_{i}\right) M^{-b_{i}} a_{i}^{b_{i}} x_{i 0}-1
$$

and if $M$ is a solution to (3.1), then

$$
\begin{aligned}
& \sum_{i \in N}\left(1-b_{i}\right) M^{-b_{i}} a_{i}^{b_{i}} x_{i 0}-1 \\
& \quad \leq \max _{i}\left(1-b_{i}\right) \sum_{i \in N} M^{-b_{i}} a_{i}^{b_{i}} x_{i 0}-1=\max _{i}\left(1-b_{i}\right)-1<0 .
\end{aligned}
$$

Thus, the conditions of Lemma 4.3 are satisfied and the required concavity of $F_{0}$ follows. 


\section{Universal bounds for asset prices}

By Proposition 3.1, the state price densities (SPDs) by themselves do lie between the maximal and minimal "homogeneous" state price densities. Depending on the state of the economy, the SPDS are closer to the representative SPD of the agent dominating in this state. But asset prices are expectations of state price densities multiplied by dividends, and the expectation of the maximum of a family of random variables is always larger than the maximum of the expectations. In particular,

$$
E\left[\max _{i} \delta_{i}^{t} W_{t}^{-\gamma_{i}}\right]>\max _{i} E\left[\delta_{i}^{t} W_{t}^{-\gamma_{i}}\right] .
$$

Proposition 3.1 implies that the price of a risk free bond satisfies

$$
B^{\mathrm{F}}(0, t)=E\left[M_{t}\right] \leq E\left[\max _{i} \delta_{i}^{t} W_{t}^{-\gamma_{i}}\right]
$$

but Dumas' conjecture is the much stronger statement

$$
E\left[M_{t}\right] \leq \max _{i} E\left[\delta_{i}^{t} W_{t}^{-\gamma_{i}}\right]
$$

When risk aversions are homogeneous, the maximum $\max _{i} \delta_{i}^{t} W_{t}^{-\gamma_{i}}$ is always attained by the agent with the largest discount factor. When risk aversions are heterogeneous, the maximum is attained by different agents in different states. This is the source of the problem.

We will need a

Definition 5.1 Let

$$
M_{\mathrm{h} t}(\delta, \gamma)=\delta^{t} W_{t}^{-\gamma}
$$

be the state price density process in the homogeneous economy populated solely by one agent with discount factor $\delta$ and risk aversion $\gamma$. Similarly, for any asset with a dividend process $\mathbf{D}$,

$$
P_{\mathrm{h} t}^{(\mathbf{D})}(\delta, \gamma)=E_{t}\left[\sum_{\tau=1}^{T-t} \frac{M_{\mathrm{h}, t+\tau}}{M_{\mathrm{h} t}}(\delta, \gamma) D_{t+\tau}\right]
$$

is the asset price in the corresponding homogeneous economy. In particular, $B_{\mathrm{h}}^{\mathrm{F}}\left(t_{1}, t_{2}\right)(\delta, \gamma)$ is the homogeneous bond price.

The following theorem clarifies Dumas' conjecture.

Theorem 5.2 We have:

- If $\gamma_{i} \geq 1$ for all $i=1, \ldots, n$ then

$$
P_{t}^{\mathbf{D}} \leq \max _{i} P_{\mathrm{h} t}^{\mathbf{D}}\left(\delta_{i}, \gamma_{i}\right)
$$


- If $\gamma_{i} \leq 1$ for all $i=1, \ldots, n$ then

$$
P_{t}^{\mathbf{D}} \geq \min _{i} P_{\mathrm{h} t}^{\mathbf{D}}\left(\delta_{i}, \gamma_{i}\right)
$$

If not all risk aversions are identical and $W_{t}$ is not constant, then all inequalities above are strict.

Proof For simplicity, we prove the result only for $t=0$. The general case follows by the same arguments with the help of Proposition 3.1.

Let $\gamma_{i} \leq 1$ for all $i$. By Proposition 4.5, $F_{0}$ is convex. Consider now the extended probability space $\Omega \oplus \cdots \oplus \Omega$ consisting of $T$ identical copies of $\Omega$ and endowed with probability measure

$$
d P^{T}=\frac{1}{T} d P \oplus \cdots \oplus d P
$$

where $d P$ is the original probability measure on $\Omega$. Then, each process $\mathbf{A}=$ $\left(A_{1}, \ldots, A_{T}\right)$ can be viewed as a random variable on the extended probability space and

$$
E^{T}[\mathbf{A}]=\frac{1}{T} \sum_{t=1}^{T} E\left[A_{t}\right] .
$$

Proposition 3.1 means that

$$
\mathbf{M}=F\left(\mathbf{M}_{\mathrm{h}}\left(\delta_{1}, \gamma_{1}\right), \ldots, \mathbf{M}_{\mathrm{h}}\left(\delta_{n}, \gamma_{n}\right)\right)
$$

where $\mathbf{M}=\left(M_{t}\right)$ is the equilibrium state price density process and $F=F_{0}$. Obviously, $F$ is homogeneous of degree one and therefore

$$
\mathbf{D M}=F\left(\mathbf{D M}_{\mathrm{h}}\left(\delta_{1}, \gamma_{1}\right), \ldots, \mathbf{D M}_{\mathrm{h}}\left(\delta_{n}, \gamma_{n}\right)\right)
$$

Using Proposition 4.5, Jensen's inequality and the estimate of Proposition 3.1, we get

$$
\begin{aligned}
T^{-1} P_{0}^{\mathbf{D}} & =T^{-1} \sum_{t=1}^{T} E\left[D_{t} M_{t}\right]=E^{T}[\mathbf{D M}] \\
& =E^{T}\left[F\left(\mathbf{D} \mathbf{M}_{\mathrm{h}}\left(\delta_{1}, \gamma_{1}\right), \ldots, \mathbf{D M}_{\mathrm{h}}\left(\delta_{n}, \gamma_{n}\right)\right)\right] \\
& \geq F\left(E^{T}\left[\mathbf{D} \mathbf{M}_{\mathrm{h}}\left(\delta_{1}, \gamma_{1}\right)\right], \ldots, E^{T}\left[\mathbf{D} \mathbf{M}_{\mathrm{h}}\left(\delta_{n}, \gamma_{n}\right)\right]\right) \\
& =F\left(T^{-1} P_{\mathrm{h} 0}^{\mathbf{D}}\left(\delta_{1}, \gamma_{1}\right), \ldots, T^{-1} P_{\mathrm{h} 0}^{\mathbf{D}}\left(\delta_{n}, \gamma_{n}\right)\right) \\
& \geq T^{-1} \min _{i} P_{\mathrm{h} 0}^{\mathbf{D}}\left(\delta_{i}, \gamma_{i}\right) .
\end{aligned}
$$

The proof for the case $\gamma_{i} \geq 1$ is completely similar and obtained by reversing all inequalities.

It remains to prove that the inequalities are strict. We only treat the case when $\gamma_{i} \geq 1$ for all $i$ and $t=0$. The other cases are completely analogous. Suppose that 
there is an identity in (5.1) and risk aversions are heterogeneous. That is, there exist $\left(b_{i}, \delta_{i}\right), i=1, \ldots, n$ such that $b_{i}=\gamma_{i}^{-1}$ are heterogeneous and

$$
\begin{aligned}
E^{T} & {\left[F\left(\mathbf{D M}_{\mathrm{h}}\left(\delta_{1}, \gamma_{1}\right), \ldots, \mathbf{D} \mathbf{M}_{\mathrm{h}}\left(\delta_{n}, \gamma_{n}\right)\right)\right] } \\
& =F\left(E^{T}\left[\mathbf{D M}_{\mathrm{h}}\left(\delta_{1}, \gamma_{1}\right)\right], \ldots, E^{T}\left[\mathbf{D M}_{\mathrm{h}}\left(\delta_{n}, \gamma_{n}\right)\right]\right) .
\end{aligned}
$$

By Lemma 4.2, identity (5.2) implies that $F$ is affine linear on the convex hull $H$ of $\operatorname{supp}(X)$ with

$$
X=\left(X_{1}, \ldots, X_{n}\right)=\left(\mathbf{D M}_{\mathrm{h}}\left(\delta_{1}, \gamma_{1}\right), \ldots, \mathbf{D M}_{\mathrm{h}}\left(\delta_{n}, \gamma_{n}\right)\right) .
$$

By Lemma 4.3, (2), this is only possible if the function

$$
G\left(x_{1}, \ldots x_{n}, y\right)=\sum_{i \in N} y^{1-b_{i}} x_{i}^{b_{i}}
$$

is affine linear on the convex hull $H_{1}$ of $\operatorname{supp}\{(X, F(X))\}$. Therefore, each function $y^{1-b_{i}} a_{i}^{b_{i}}$ should be affine linear on this convex hull. By Lemma 4.4, this is only possible if

$$
h_{i} F(h)^{-1}=g_{i} F(g)^{-1}
$$

for all $h, g \in H$ and all $i=1, \ldots, n$. Thus, all vectors $h \in \operatorname{supp}(X)$ should be colinear and there exist a scalar random variable $z$ and a vector $h \in \mathbb{R}^{n}$ such that

$$
\left(\mathbf{D M}_{\mathrm{h}}\left(\delta_{1}, \gamma_{1}\right), \ldots, \mathbf{D} \mathbf{M}_{\mathrm{h}}\left(\delta_{n}, \gamma_{n}\right)\right)=z h
$$

almost surely. Let for simplicity $\gamma_{1} \neq \gamma_{2}$. The above identity implies that

$$
\frac{\mathbf{M}_{\mathrm{h}}\left(\delta_{1}, \gamma_{1}\right)}{\mathbf{M}_{\mathrm{h}}\left(\delta_{2}, \gamma_{2}\right)}=\frac{\mathbf{D} \mathbf{M}_{\mathrm{h}}\left(\delta_{1}, \gamma_{1}\right)}{\mathbf{D M}_{\mathrm{h}}\left(\delta_{2}, \gamma_{2}\right)}=\frac{h_{1}}{h_{2}}
$$

is a constant. But $\mathbf{M}_{\mathrm{h}}(\delta, \gamma)=\left(\delta^{t} W_{t}^{-\gamma}\right)$, and the last identity is impossible if $W_{t}$ is random. This gives a contradiction.

When risk aversions are homogeneous and equal $\gamma$, the aggregator function has a simple structure; we get $M_{t}=\left(\sum_{i=1}^{n}\left(\delta_{i}^{t} W_{t}^{-\gamma}\right)^{b} x_{i 0}\right)^{1 / b}$, with $b=\gamma^{-1}$. In particular, when all risk aversions equal one, the aggregator function is linear and state price densities are simply linear combinations of representative state price densities. When risk aversions are heterogeneous, the structure becomes quite complicated, but risk aversion equal to one remains a natural threshold for convexity/concavity.

The bounds of Theorem 5.2 are important for empirical applications because they are universal and hold for all time periods. For example, it is known that the real world price-dividend ratios are highly volatile. The bounds of Theorem 5.2 show that the size of the interval in which the P-D ratios fluctuate depends on the heterogeneity. In particular, one can use real data and Theorem 5.2 to understand the strength of heterogeneity in the real world.

Finally, we give a general result stating that Dumas' conjecture is wrong in general. 
Proposition 5.3 Suppose that $\mathbf{W}=\left(W_{t}\right)$ is not almost surely constant. Fix an asset with dividend process $\mathbf{D}$. Then, for any collection of heterogeneous risk aversions $\gamma_{1}, \ldots, \gamma_{n}$ such that either $\gamma_{i} \geq 1$ for all $i$ or $\gamma_{i} \leq 1$ for all $i$, there exists a collection of discount factors $\delta_{1}, \ldots, \delta_{n}$ such that one of the inequalities

$$
\min _{i} P_{\mathrm{h} t}^{\mathbf{D}}\left(\delta_{i}, \gamma_{i}\right) \leq P_{t}^{\mathbf{D}} \leq \max _{i} P_{\mathrm{h} t}^{\mathbf{D}}\left(\delta_{i}, \gamma_{i}\right)
$$

is violated.

Proof By construction, we can always choose a collection of $\delta_{1}, \ldots, \delta_{n}$ in $(0,1)$ such that

$$
P_{\mathrm{h} t}^{\mathbf{D}}\left(\delta_{i}, \gamma_{i}\right)=P_{\mathrm{h} t}^{\mathbf{D}}\left(\delta_{j}, \gamma_{j}\right)
$$

for all $i, j$. In this case (5.4) turns into an identity and Theorem 5.2 implies that (5.4) cannot hold, since the inequalities must be strict.

\section{Risk aversion on both sides of one}

It is possible to extend the results of the previous section to the general case when the risk aversions of heterogeneous agents lie on both sides of one.

Let

$$
\bar{\gamma}:=\max \left\{1, \max _{i} \gamma_{i}\right\} \geq 1
$$

and

$$
\underline{\gamma}:=\min \left\{1, \min _{i} \gamma_{i}\right\} \leq 1
$$

We can rewrite the equation for $M$ in the form

$$
\sum_{i \in N}\left(M_{t}^{1 / \underline{\gamma}}\right)^{-b_{i} \underline{\gamma}}\left(\delta_{i}^{t / \underline{\gamma}} W_{t}^{-\gamma_{i} / \underline{\gamma}}\right)^{b_{i}} \underline{\underline{\gamma}} x_{i 0}=1,
$$

or

$$
\sum_{i \in N}\left(M_{t}^{1 / \bar{\gamma}}\right)^{-b_{i} \bar{\gamma}}\left(\delta_{i}^{t / \bar{\gamma}} W_{t}^{-\gamma_{i} / \bar{\gamma}}\right)^{b_{i} \bar{\gamma}} x_{i 0}=1 .
$$

By definition of $\gamma$, we have $b_{i} \gamma \leq 1$ for all $i$ and, similarly, $b_{i} \bar{\gamma} \geq 1$ for all $i$. Therefore, the same arguments as above apply. The only problem is for assets with multiperiod payoffs, because then powers of the number of periods arise. For simplicity, we formulate the result only for zero coupon risk free bonds.

Proposition 6.1 The price $B^{\mathrm{F}}(t, t+\tau)$ of a zero coupon risk free bond satisfies

$$
\min _{i}\left(E_{t}\left[\delta_{i}^{\tau / \bar{\gamma}}\left(\frac{W_{t+\tau}}{W_{t}}\right)^{-\gamma_{i} / \bar{\gamma}}\right]\right)^{\bar{\gamma}}
$$




$$
\leq B^{\mathrm{F}}(t, t+\tau) \leq \max _{i}\left(E_{t}\left[\delta_{i}^{\tau / \underline{\gamma}}\left(\frac{W_{t+\tau}}{W_{t}}\right)^{-\gamma_{i} / \underline{\gamma}}\right]\right)^{\underline{\gamma}},
$$

and all inequalities are strict if risk aversions are heterogeneous and $\mathbf{W}$ is not almost surely constant.

Proof For brevity, we write down the argument only for prices at time zero. Hölder's inequality and Theorem 5.2 together yield

$$
E\left[M_{t}\right] \leq\left(E\left[M_{t}^{1 / \underline{\gamma}}\right]\right)^{\underline{\gamma}} \leq \max _{i}\left(E\left[\delta_{i}^{t / \underline{\gamma}} W_{t}^{-\gamma_{i} / \underline{\gamma}}\right]\right)^{\underline{\gamma}}
$$

The same argument applies to the lower bounds.

\section{References}

1. Abel, A.B.: Asset pricing under habit formation and catching up with the Joneses. Am. Econ. Rev. 80(2), 38-42 (1990)

2. Benninga, S., Mayshar, J.: Heterogeneity and option pricing. Rev. Deriv. Res. 4, 7-27 (2000)

3. Chan, Y.L., Kogan, L.: Catching up with the Joneses: Heterogenous preferences and the dynamics of asset prices. J. Polit. Econ. 110, 1255-1285 (2002)

4. Constantinides, G.M.: Intertemporal asset pricing with heterogeneous consumers and without demand aggregation. J. Bus. 55, 253-267 (1982)

5. Constantinides, G.M.: Habit formation: a resolution of the equity premium puzzle. J. Polit. Econ. 98, 519-543 (1990)

6. Dumas, B.: Two-person dynamic equilibrium in the capital market. Rev. Financ. Stud. 2, 157-188 (1989)

7. Gollier, C., Zeckhauser, R.J.: Aggregation of heterogeneous time preferences. J. Polit. Econ. 113, 878-896 (2005)

8. Lengwiler, Y.: Heterogenous patience and the term structure of real interest rates. Am. Econ. Rev. 95, 890-896 (2005)

9. Malamud, S.: Long run forward rates and long yields of bonds and options in heterogeneous equilibria. Finance Stoch. 12, 248-268 (2008)

10. Rubinstein, M.: An aggregation theorem for securities markets. J. Financ. Econ. 1, 225-244 (1974)

11. Wang, J.: A model of intertemporal asset pricing under asymmetric information. Rev. Financ. Stud. 60, 249-282 (1993)

12. Wang, J.: A model of competitive stock trading volume. J. Polit. Econ. 102, 127-168 (1994)

13. Wang, J.: The term structure of interest rates in a pure exchange economy with heterogenous investors. J. Financ. Econ. 41, 75-110 (1996) 